\title{
Stochastic Modeling of A Non Maintained System With Two Stages of Deterioration
}

\author{
Ibrahim Yusuf ${ }^{1}$, Saminu I. Bala ${ }^{2}$ and U.A.Ali ${ }^{3}$ \\ Department of mathematical Sciences, Bayero University, Kano, Nigeria \\ ${ }^{3}$ Department of Mathematics, Faculty of Science, Usmanu Danfodio University, \\ Sokoto, Nigeria \\ Email: ㅎrahimyusif@yahoo.com, saminub@yahoo.com, ubahamad@yahoo.co.uk
}

\begin{abstract}
This paper presents the reliability and mean time to failure estimation of a deteriorating system that deteriorates with time. The system is two unit active parallel system where the units operates simultaneously. In this study maintenance is not allowed to the system. Laplace transforms is being utilize to solve the mathematical model developed. Expressions for reliability and Mean time to failure (MTTF) have been computed. The objective is to see the effect of deterioration rates on system reliability and MTTF of a non maintained system. It also the objective of this study to see the effect of time on system reliability. Graphical study of the system through reliability and MTTF is obtained to highlight important results. The results have shown that reliability and MTTF of non maintained system decrease as time and deterioration rates increase. Models developed in this paper are important to engineers, maintenance managers, and plant management for proper maintenance decision and for safety of the system as a whole.
\end{abstract}

Keywords: Reliability, MTTF, Laplace transformation, deterioration

\section{Introduction}

Systems are subject to deterioration meaning that in course of time their condition falls from higher to lower, and possibly even to unacceptable, levels. Obviously, the condition level and the performance of the system are related. Generally the lower the condition level the worse the performance of the system. Maintenance 
actions such as inspection, local repair and replacement should be done to retain the system in or restore it to an acceptable operating condition.

Many technical systems are subjected to aging and degradation during their lifetime. Most of these systems are repairable. Deterioration has a great influence not only on inventory management, but on every area of production where items are stocked or forced to wait due to technical matters, variability or disruptions / stochastic influences in the production process. For a deteriorating system, it is reasonable to assume that the successive operating times of the system after repairs are stochastically decreasing and the consecutive repair times of the system after failures are stochastically increasing.

Because deterioration is uncertain over time, it should ideally be represented as a stochastic process. Most engineering assets experience some kind of deterioration before failing. Asset failures can be predicted based on these deterioration processes which are revealed by various deterioration indicators.

Studies on stochastic analysis on systems can be found in Ahmad and Singh [1], Ahmad et al [2], Gupta et al [3], kishan and Kumar [4], Mokaddis et al [5], Ritu et al [6], Singh and Singh [7], Sridharan and Kalyan [8],Mathew et al [9] studies the reliability of continuous casting plant system operating with full installed capacity using semi Markov processes and regenerative point techniques. While research on maintenance and replacement of deterioration system can be found in Chen and Wu [10], Pierskalla and Voelker [11], Valderz and Feldman [12], Wang [13] and Abdel-Hameed [14].

This paper studied reliability and mean time to failure (MTTF) estimation of two unit active parallel system and the effect of the time and deterioration rates on reliability and the effect of deterioration rates on MTTF using Laplace transformation.

\section{Model Formulation}

\subsection{Assumption}

1. State of the system can be: Perfect $\left(S_{1}\right)$, Minor deterioration $\left(S_{2}\right)$, Major deterioration $\left(S_{3}\right)$, Failure $\left(S_{4}\right)$

2. At any given time $t$ the system is either in the operating state, deteriorating state or in the failed state.

3. The units operate simultaneously

4. State $S_{4}$ can be access from the previous state

5. The state of the system changes as time progresses

6. System/units work in $S_{1}, S_{2}, S_{3}$ and fail in $S_{4}$

7. the deteriorate stages can be minor or major 


\subsection{Notations and Nomenclature}

$\lambda_{12}, \lambda_{13}, \lambda_{23}$ : Deterioration rate

$\lambda_{14}$ : Failure rate of the system while in $S_{1}$

$\lambda_{24}$ : Failure rate of the system while in $S_{2}$

$\lambda_{34}:$ Failure rate of the system while in $S_{3}$

$R(t)$ : Reliability of the system at time $t$

MTTF: Mean time to failure of the system
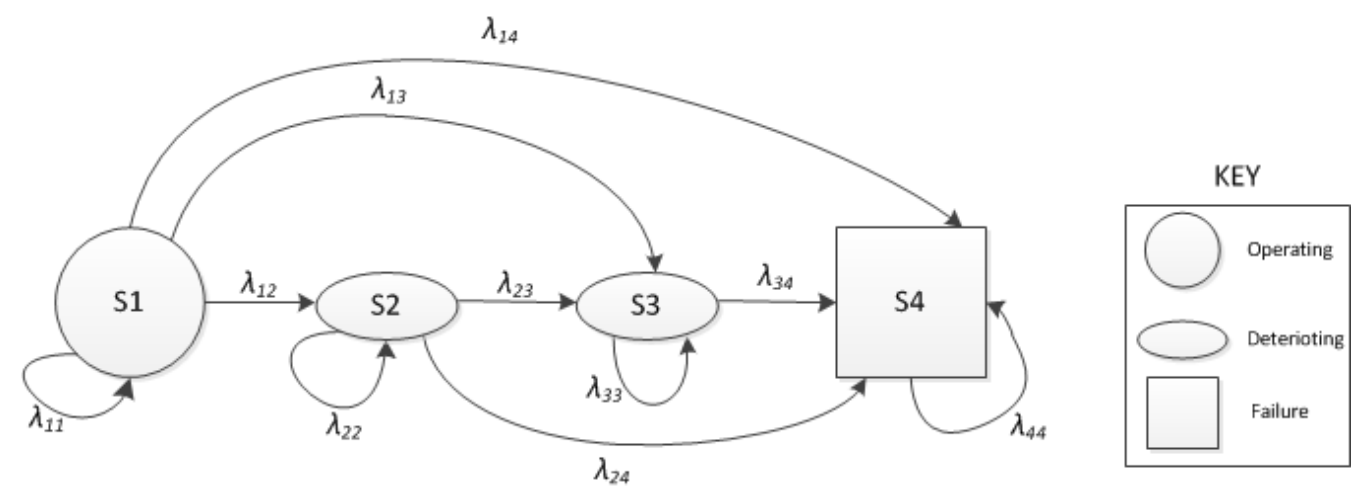

Fig 1: Transition diagram of non maintained system

From fig. 1 above, we obtained the following first order differential equations

$$
\begin{aligned}
\frac{d p_{1}(t)}{d t}= & -\left(\lambda_{12}+\lambda_{13}+\lambda_{14}\right) P_{1}(t) \\
\frac{d p_{2}(t)}{d t} & =\lambda_{12} P_{1}(t)-\left(\lambda_{23}+\lambda_{24}\right) P_{2}(t) \\
\frac{d p_{3}(t)}{d t} & =\lambda_{13} P_{1}(t)+\lambda_{23} P_{2}(t)-\lambda_{34} P_{3}(t) \\
\frac{d P_{4}(t)}{d t} & =\lambda_{14} P_{1}(t)+\lambda_{24} P_{2}(t)+\lambda_{34} P_{3}(t)
\end{aligned}
$$

Subject to the condition $P_{1}(0)=1, P_{2}(0)=P_{3}(0)=P_{4}(0)=0$

Taking the Laplace transformation of (1) we have

$$
\begin{aligned}
& P_{1}(s)=\frac{1}{s+a} \\
& P_{2}(s)=\frac{-\lambda_{12}}{(a-b)(s+a)}+\frac{\lambda_{12}}{(a-b)(s+b)} \\
& P_{3}(s)=\frac{\lambda_{12}}{(a-c)(s+c)}-\frac{\lambda_{12}}{(a-c)(s+a)}+\frac{\lambda_{12} \lambda_{23}}{(a-b)(b-c)(s+c)}- \\
& \frac{\lambda_{12} \lambda_{23}}{(a-b)(b-c)(s+b)}-\frac{\lambda_{12} \lambda_{23}}{(a-b)(a-c)(s+c)}+\frac{\lambda_{12} \lambda_{23}}{(a-b)(a-c)(s+a)}
\end{aligned}
$$


Where $a=\lambda_{12}+\lambda_{13}+\lambda_{14}, b=\lambda_{23}+\lambda_{24}$, and $c=\lambda_{34}$

From the inverse Laplace transformation of (2) we have

$$
\begin{aligned}
P_{1}(t)= & e^{-\left(\lambda_{12}+\lambda_{13}+\lambda_{14}\right) t} \\
P_{2}(t)= & \frac{\lambda_{12}}{\left(\lambda_{2}+\lambda_{13}+\lambda_{14}-\lambda_{23}-\lambda_{24}\right)} e^{-\left(\lambda_{23}+\lambda_{24}\right) t}-\frac{\lambda_{12}}{\left(\lambda_{2}+\lambda_{13}+\lambda_{14}-\lambda_{23}-\lambda_{24}\right)} e^{-\left(\lambda_{12}+\lambda_{13}+\lambda_{14}\right) t} \\
P_{3}(t)= & \frac{-\lambda_{12} \lambda_{23}}{\left(-\lambda_{34}+\lambda_{23}+\lambda_{24}\right)\left(\lambda_{12}+\lambda_{13}+\lambda_{14}-\lambda_{23}-\lambda_{24}\right)} e^{-\left(\lambda_{23}+\lambda_{24}\right) t} \\
& -\frac{\left(\lambda_{13} \lambda_{12}+\lambda_{13}^{2}+\lambda_{13} \lambda_{14}-\lambda_{13} \lambda_{23}-\lambda_{13} \lambda_{24}-\lambda_{23} \lambda_{2}\right)}{\left(-\lambda_{34}+\lambda_{12}+\lambda_{13}+\lambda_{14}\right)\left(\lambda_{12}+\lambda_{13}+\lambda_{14}-\lambda_{23}-\lambda_{24}\right)} e^{-\left(\lambda_{12}+\lambda_{13}+\lambda_{14}\right) t} \\
& +\frac{\left(\lambda_{23} \lambda_{12}-\lambda_{13} \lambda_{34}+\lambda_{13} \lambda_{23}+\lambda_{13} \lambda_{24}\right)}{\left(-\lambda_{34}+\lambda_{23}+\lambda_{24}\right)\left(-\lambda_{34}+\lambda_{12}+\lambda_{13}+\lambda_{14}\right)} e^{-\lambda_{34} t} \\
R(t)= & P_{1}(t)+P_{2}(t)+P_{3}(t) \\
\text { M.T.T.F }= & \int_{0}^{\infty} R(t) d t=\frac{\lambda_{23} \lambda_{34}+\lambda_{24} \lambda_{34}+\lambda_{12} \lambda_{34}+\lambda_{13} \lambda_{24}+\lambda_{13} \lambda_{23}+\lambda_{23} \lambda_{12}}{\lambda_{34}\left(\lambda_{12}+\lambda_{13}+\lambda_{14}\right)\left(\lambda_{23}+\lambda_{24}\right)}
\end{aligned}
$$

\section{Results}

Fig. 2 is the relationship between reliability and time $t$

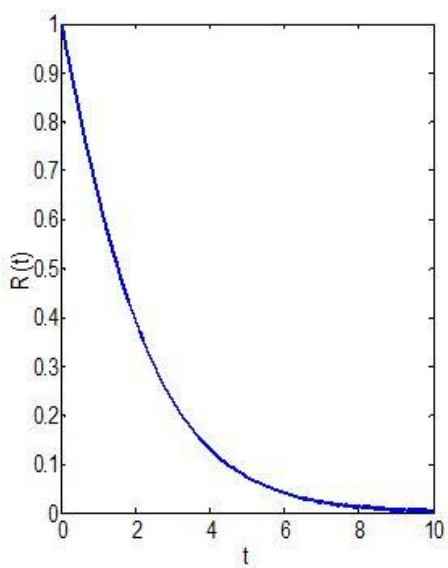

fig. 2 plot of $R(t)$ against time $t$ 

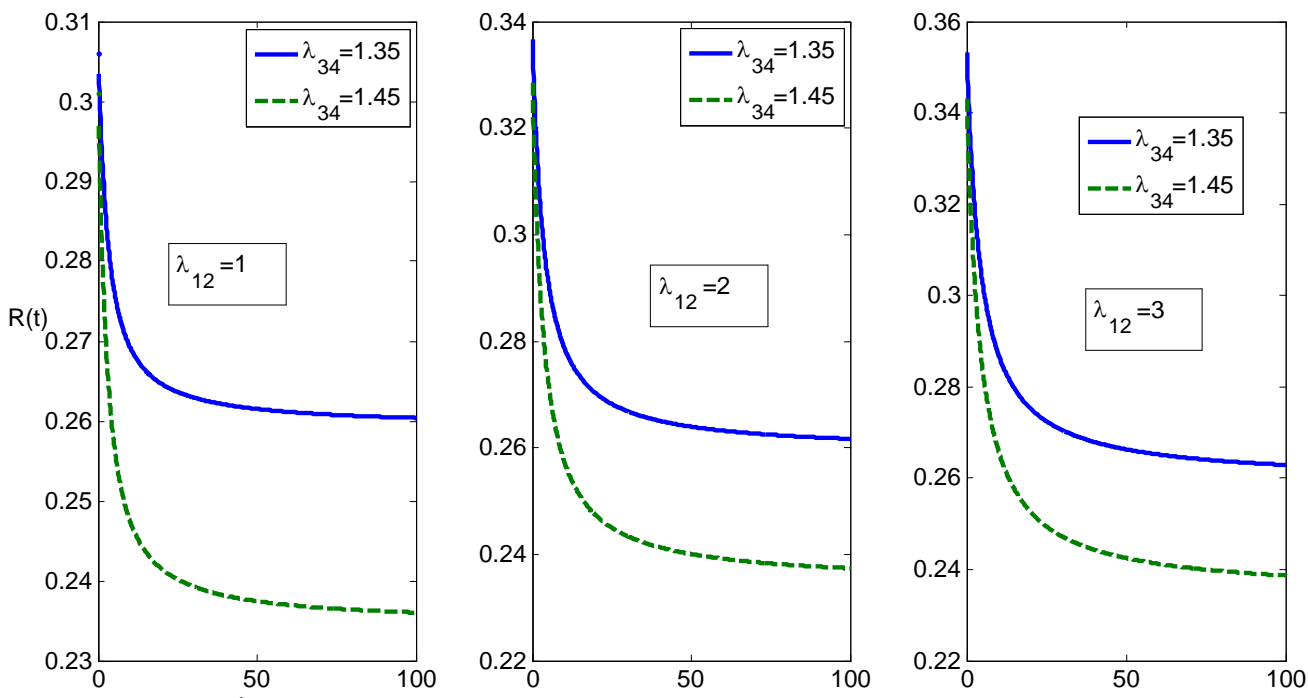

Fig. 3 effect of $\lambda_{13}$ on system reliability $R(t)$
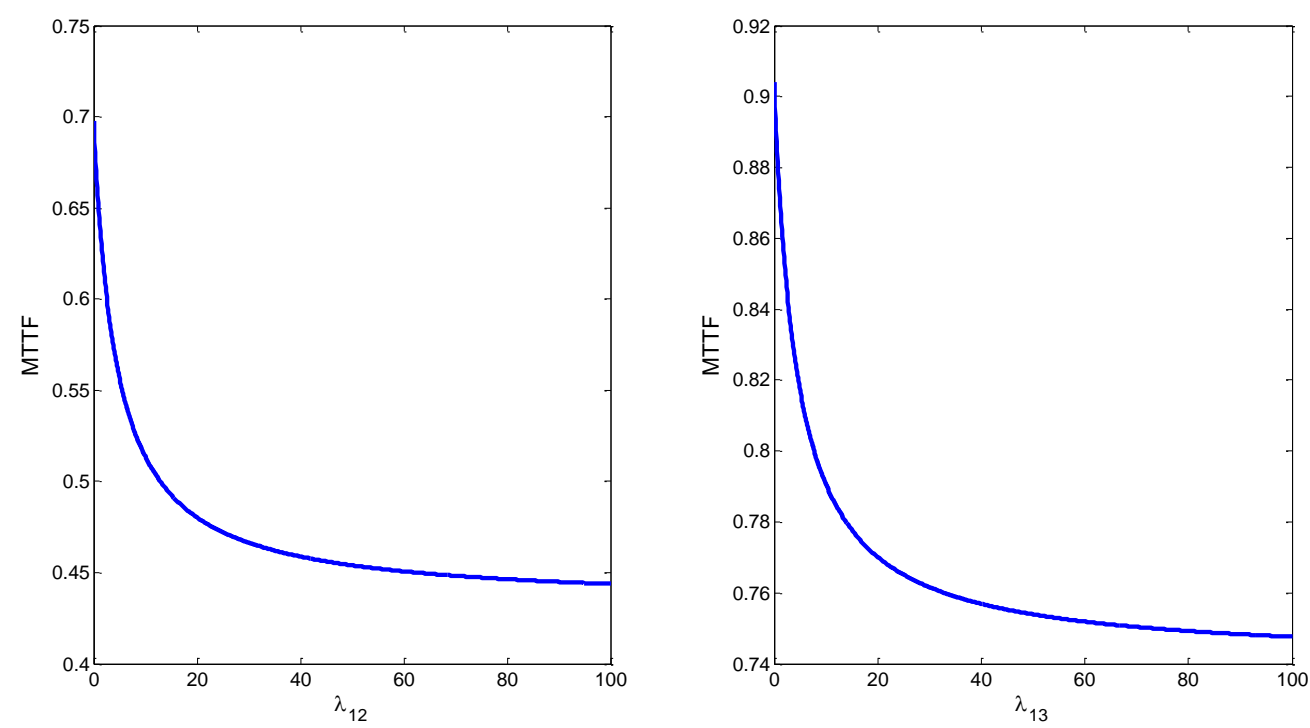

Fig. 4 effect of deterioration rates on MTTF

\section{Conclusion}

In this study,explicit expression for system reliability and mean time to failure of a non maintained system was developed. It has been found that reliability decreases with time and deterioration rates. That Reliability decreases with time and deterioration rate is consistent according the simulation of the model. It was 
found from the simulation that MTTF decreases with increase in deterioration rates.

\section{Acknowledgement}

The authors acknowledged the effort of the editor and reviewers toward evaluation of this article.

\section{References}

[1] Ahmad, A. and Singh, L.; 2009. Stochastic analysis of a two Identical Unit Cold Standby System with maximum repair times and Correlated busy and idle times of the Operator cum repairman. J. Informatics and Mathematical Sciences, 2 \& 3: 147-155.

[2] Ahmad, A., Singh, L., and G. Varshney, 2010. Stochastic analysis and Inference on two units Standby System with three Phases of repair. Int, J. Agricult. Stat., 6(2): 629-636.

[3] Gupta, P.P and Arvind Kumar, 1986. Reliability and MTTF analysis of non repairable Parallel redundant complex System under hardware and human failures. Microelect. Reliab.; 26: 229-234.

[4] Kishan, R and Kumar, M.,2010. Stochastic analysis of a complex system with correlated working and rest period of repairman. Journal of Reliability and Statistical Studies, 3(2): 61-72.

[5] Mokaddis, G.S, El-Sherbeny M.S and Al-Esayeh E, 2009. Stochastic Behavior of Redundant Complex System with two types of failures. J.Math. Stat.,5(2): 112-117.

[6] Ritu Gupta, S.K. Mittal and C.M. Batra, 2006. Stochastic analysis of a Compound Redundant System involving human failure. J. Math. Stat. 2: 407413.

[7] Singh, S.K and R.P. Singh, 1989. Stochastic analysis of a Complex System with two types of repair facilities and Patience time for repair. IJOMAS, 5(1): 43-56.

[8] Sridharan,V and Kalyan,T.V, 2002.Stochastic analysis of a non identical two unit Parallel System with Common cause failure using GERT technique. Information and Management Sciences, 13(1): 49-57.

[9] Matthew, A.G, S.M. Rizwan, M.L. Majumder, K. P. Ramachandran and G. Taneja, 2011. Reliability analysis of an identical two unit Parallel Continuous Casting Plant Systm operative with full Installed Capacity. Int. J. of Performability Engineering, 7(2): 179-185.

[10] Chen, A., \& Wu, R. S. (2007). Real-time healthy index and dynamic preventive maintenance policy for equipment under aging Markovian 
deterioration. International Journal of Production Research, 15(1), 33513379.

[11] Pierskalla, W. P., \& Voelker, J. A. (1976). A survey of maintenance models: The control and surveillance of deteriorating systems. Naval Research Logistic Quarterly, 23, 353-388.

[12] Valderz-Flores, C., \& Feldman, R. M. (1989). A survey of preventive maintenance models for stochastically deteriorating single-unit system. Naval Research Logistic Quarterly, 36, 419-446.

[13] Wang, H. (2002). A survey of maintenance policies of deteriorating systems. European Journal of Operation Research, 139, 469-489.

[14] Abdel-Hameed, M. (1984a). Deterioration processes." Semi-Markov Models: Theory and Applications, J. Janssen, ed., New York. Plenum Publishing Corporation, 231-252. 